\title{
Chiroptical Properties of Oligomers of $m$-Methylphenyl Isocyanate Bearing an Optically Active End-Group
}

\author{
Katsuhiro Maeda, Masashi Matsuda, Tamaki NaKano, \\ and Yoshio Окамото* \\ Department of Applied Chemistry, School of Engineering, Nagoya University, \\ Furo-cho, Chikusa-ku, Nagoya 464-01, Japan
}

(Received June 16, 1994)

\begin{abstract}
Oligomerization of $m$-methylphenyl isocyanate ( $m \mathrm{MePI})$ with lithium amide of $(S)-(-)-2-($ methoxymethyl)pyrrolidine (MMP) was carried out to obtain oligomers containing a chiral residue at the initial chain end ( $\alpha$-end). The oligomers of degree of polymerization (DP) less than eighteen were separated in terms of DP and the specific rotation of each oligomer was estimated. The oligomers showed the high optical rotation which is ascribed to the structure prevailing in one-screw sense. The specific rotation of the oligomers increased up to DP of twelve and then decreased with an increase in DP. This suggests that the DP corresponding to the average persistence length of single handed helix may be larger than twelve. The specific rotation of the polymers was inversely proportional to the molecular weight. The optical activity of the polymer greatly increased with a decrease in temperature, whereas that of the trimer was not influenced by temperature.
\end{abstract}

KEY WORDS Oligomer / Aromatic Isocyanate / Helix / Optically Active

Polymer / Anionic Polymerization /

Poly(alkyl isocyanate)s have a rigid rod-like main chain based on the structure of 1-nylon ${ }^{1,2}$ and have extensively been studied from the viewpoints of conformation. Polyisocyanates with optically active side groups can provide important information on a helical structure of the polymers. ${ }^{3-8}$ It has been pointed out that poly(aromatic isocyanate)s have much less stiff conformation than that of poly(alkyl isocyanate)s. ${ }^{1}$ However, we recently reported that achiral aromatic isocyanates ${ }^{9}$ as well as alkyl isocyanates ${ }^{10}$ can afford optically active polymers in the polymerization with chiral anionic initiators such as lithium alkoxides or amides. The obtained polymers showed the optical activity based on the prevailing helicity of a main chain induced by a chiral initiator residue attached to the initial chain end ( $\alpha$-end). The optical activity of the polyisocyanates decreases with an increase in molecular weight of the polymers. This is because the one-handed helical structure induced by the chiral initiator residue can persist only in a rather short range from the $\alpha$-end.

In the present study, we synthesized the oligomer of $m$-methylphenyl isocyanate ( $m \mathrm{Me}$ PI) with a chiral fragment at the $\alpha$-end and estimated its specific rotation in order to get information on the helical structure of poly(aromatic isocyanate)s.

\section{EXPERIMENTAL}

\section{Materials}

$(S)-(-)-2-($ Methoxymethyl)pyrrolidine $[(-)$-MMP $]\left(\right.$ Merck, $\left.[\alpha]_{\mathrm{D}}-3^{\circ}\right)$ was purified by distillation over $\mathrm{CaH}_{2}$ under reduced pressure. $m \mathrm{MePI}$ (Aldrich) was distilled over

\footnotetext{
* To whom all correspondence should be addressed.
} 
$\mathrm{CaH}_{2}$ under reduced pressure just before polymerization. Tetrahydrofuran (THF) was purified by distillation from $\mathrm{Na}$ and $\mathrm{CaH}_{2}$ and then distilled again from $\mathrm{LiAlH}_{4}$ under high vacuum just before use.

\section{Preparation of Initiator Solution}

Lithium amide of (-)-MMP was prepared by adding an equimolar amount of tert-butyllithium solution in pentane to a solution of (-)-MMP in THF at room temperature.

\section{Oligomerization Procedure}

Oligomerization was carried out under a dry nitrogen atmosphere. A THF solution of $m \mathrm{MePI}$ in a glass ampule was cooled to $-98^{\circ} \mathrm{C}$. The Li-MMP solution was then added to the monomer solution with a syringe. The reaction was terminated by adding a small amount of methanol containing hydrochloric acid after $4 \mathrm{~h}$ and the reaction mixture was kept for an hour at the temperature for complete termination. The mixture was added in a large amount of methanol and the polymer precipitated was separated by filtration. The filtrate was concentrated by distillation in vacuo, and then chloroform was added to the residue. The solution was washed with water. The chloroform layer was dried over magnesium sulfate and then chloroform was removed by distillation to recover the oligomer.

\section{Fractionation of Oligomer}

Fractionation of the oligomer was performed by a high-performance liquid chromatograph (JASCO 880-PU) equipped with a JASCO 875-UV (UV) and a Shodex OR-1 (polarimeter) detectors on a silica gel column $(25 \times 0.46$ (i.d.) $\mathrm{cm})$ using a mixture of hexane and 2-propanol $(9: 1, \mathrm{v} / \mathrm{v})$ as an eluent. The structure and DP of oligomers were determined by ${ }^{1} \mathrm{H}$ NMR and FD mass spectroscopies.

\section{Measurements}

The molecular weight of the polymer was determined by GPC measurement on a TOSOH HLC 802A GPC system equipped with an RI detector and a TOSOH LS-8 light scattering detector ( $\mathrm{He}-\mathrm{Ne}$ laser with a detection angle of $5^{\circ}$ ) by using a Shodex-KF-80M GPC column (eluent: THF). Optical rotation was measured on a JASCO DIP-181 polarimeter. IR spectra were recorded using a JASCO FT-IR-7000 infrared spectrometer. Circular dichroism spectra were taken using a JASCO J-720 spectrometer. ${ }^{1} \mathrm{H}$ NMR spectra were measured at $60^{\circ} \mathrm{C}$ using a Varian VXR-500 $(500 \mathrm{MHz})$ spectrometer with tetramethylsilane as an internal standard. FD mass spectra were taken using a JEOL JMS-AX505HA instrument.

\section{RESULTS AND DISCUSSION}

Table I shows the results of the oligomerization of $m \mathrm{MePI}$ with Li-MMP in THF at $-98^{\circ} \mathrm{C}$. Molar ratios of monomer to initiator were changed from 1 to 10. Methanol-insoluble polymer was not formed at the ratios 1,3 , and 5 , but was formed at the ratio 10 . The polymer showed a large optical activity $\left([\alpha]_{365}^{25}\right.$ $\left.+1107^{\circ}\right)$. Figure 1 shows a GPC curve of oligo-I-3 (No. 3 in Table I). The number in the figure corresponds to DPs. MMP and the unimer (1-mer) show negative optical rotation, but the higher oligomers show positive optical

Table I. Oligomerization of $m \mathrm{MePI}$ with Li-MMP in THF at $-98^{\circ} \mathrm{C}^{\mathrm{a}}$

\begin{tabular}{|c|c|c|c|}
\hline \multirow[b]{2}{*}{ No. } & \multirow{2}{*}{$\begin{array}{l}{[m \mathrm{MePI}] /} \\
{[\mathrm{Li}-\mathrm{MMP}]}\end{array}$} & \multicolumn{2}{|c|}{ Yield $/ \%^{b}$} \\
\hline & & $\begin{array}{l}\text { Methanol- } \\
\text { insoluble }\end{array}$ & $\begin{array}{c}\text { Methanol- } \\
\text { soluble }\end{array}$ \\
\hline 1 & 1 & 0 & 149 \\
\hline 2 & 3 & 0 & 115 \\
\hline 3 & 5 & 0 & 102 \\
\hline $4^{c}$ & 10 & $20^{\mathrm{d}}$ & 90 \\
\hline
\end{tabular}

\footnotetext{
a $m \mathrm{MePI} 0.3 \mathrm{~g}$, THF $5 \mathrm{ml}$, time $40 \mathrm{~h}$.

b Based on $m \mathrm{MePI}$.

c $m$ MePI $1.0 \mathrm{~g}$.

d $M_{n}=6.6 \times 10^{3}, M_{w} / M_{n}=1.2,[\alpha]_{365}^{25}+1107^{\circ}$.
} 
rotation. This indicates that the positive optical rotation of the polymer is based on the prevailing helicity of main chain. The content of trimer (3-mer) was much higher in comparison with others. In the initial stage of the polymerization, the reaction seems to proceed quickly to 3 -mer and the step from

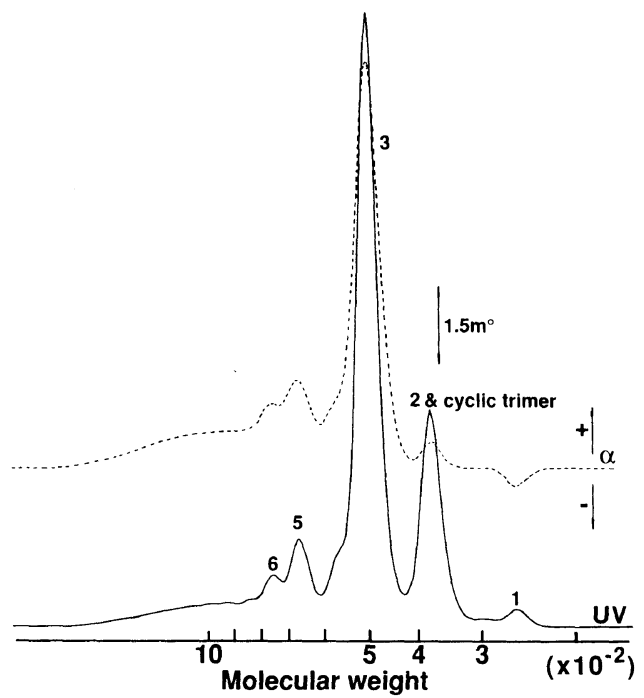

Figure 1. GPC curves of oligo-I-3 (No. 3 in Table I) measured with polarimeter (dashed curve) and UV $(270 \mathrm{~nm})$ (continuous curve) detectors. 3-mer to tetramer (4-mer) is retarded probably due to sterical reason. 4-Mer was not clearly detected. The step from 4-mer to 5-mer may be fast.

Figure 2 shows the ${ }^{1} \mathrm{H}$ NMR spectrum of the $3-\operatorname{mer}\left(M_{w}=514\right.$ by FD mass $)$. Three peaks due to the methyl groups on the phenyl groups were observed at $2.3 \mathrm{ppm}$. The other peaks in the range of $1-4 \mathrm{ppm}$ are assignable to the MMP residue at the $\alpha$-end. The intensity of each peak well agreed with the structure of the 3-mer.

Figures 3 and 4 show the chromatograms of the methanol-soluble oligomers (oligo-I-1 and 4) obtained in run No. 1 and 4 in Table I, respectively. The separation was done on a silica gel column. The elution time of the oligomers except for the 1-mer increased with an increase in DP. 4-Mer was not detected. In case of the oligomerization of $n$-butyl isocyanate, no oligomers were obtained under the same reaction conditions.

The UV and CD spectra of 1-mer, 3-mer, 5-mer, and polymer are shown in Figures 5 and 6. The UV spectral patterns of the oligomer of DP $\leqq 5$ changed drastically, but those of DP

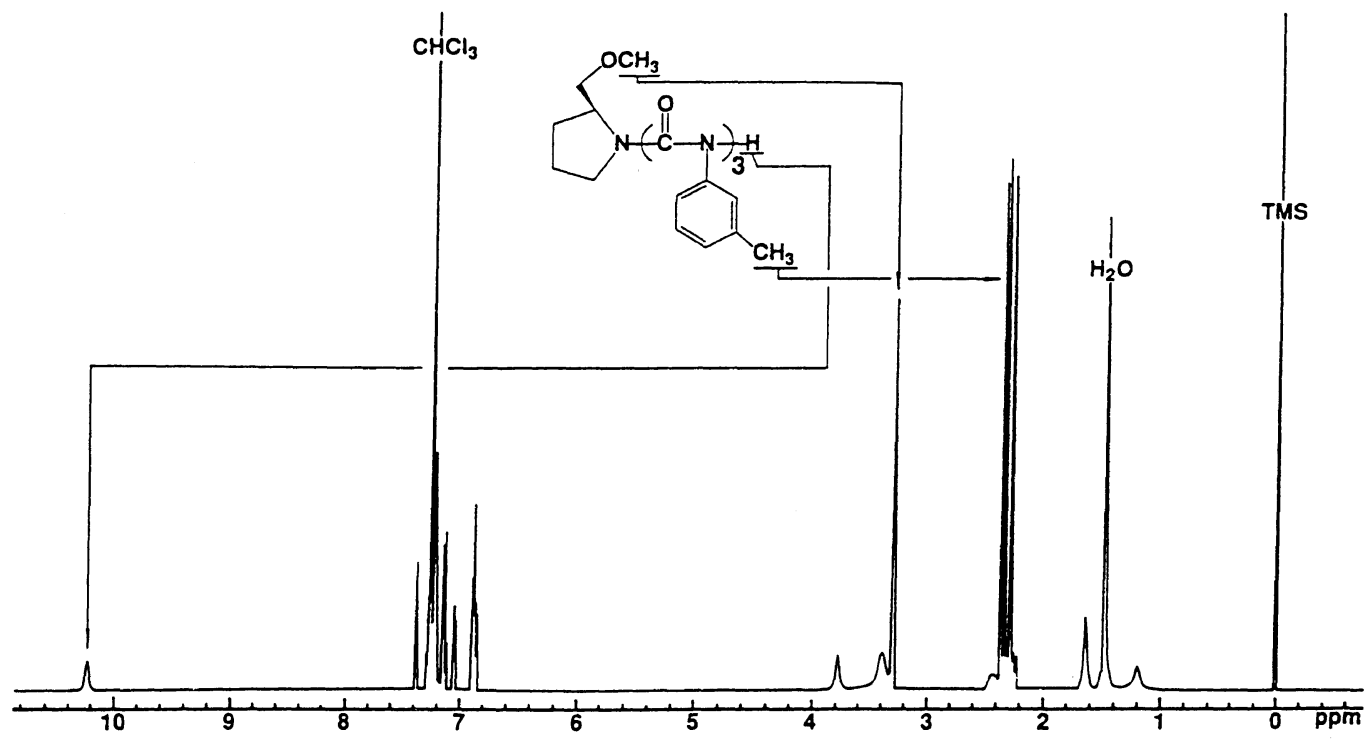

Figure 2. ${ }^{1} \mathrm{H}$ NMR spectrum of trimer in $\mathrm{CDCl}_{3}$ at $60^{\circ} \mathrm{C}(500 \mathrm{MHz})$. 


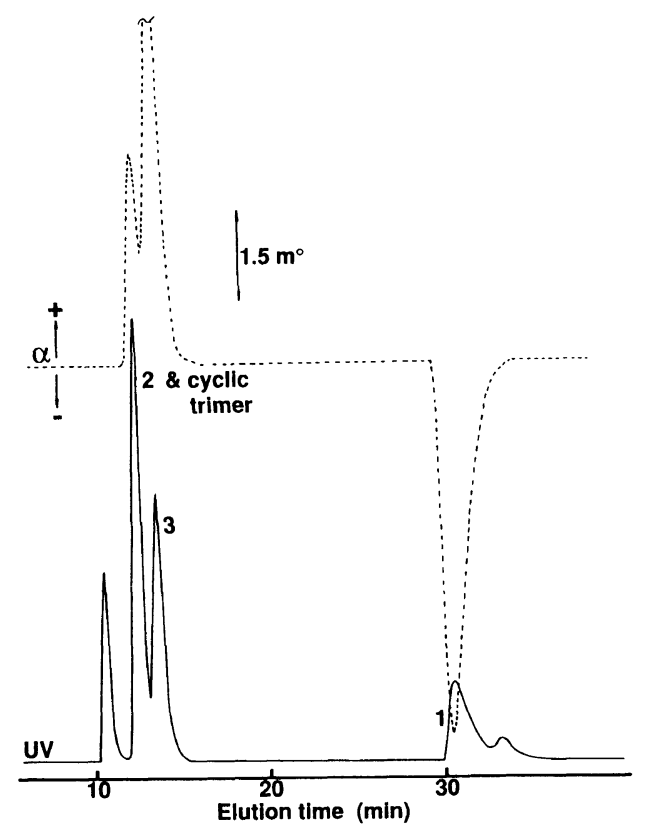

Figure 3. HPLC chromatograms of oligo-I-1 (No. 1 in Table I) (column: silica gel, $25 \times 0.46$ (i.d.) $\mathrm{cm}$; eluent: hexane-2-propanol (90: 10); $0.5 \mathrm{ml} \mathrm{min}^{-1}$ ).

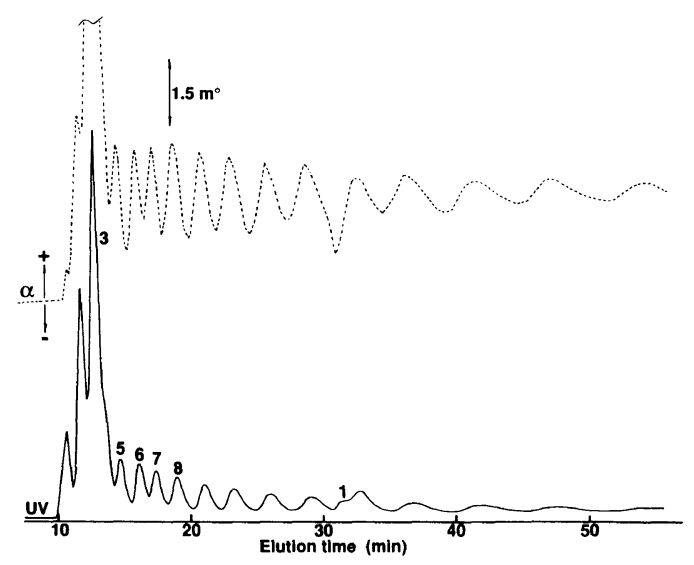

Figure 4. HPLC chromatograms of oligo-I-4 (No. 4 in Table I) (column: silica gel, $25 \times 0.46$ (i.d.) $\mathrm{cm}$; eluent: hexane-2-propanol $\left.(90: 10) ; 0.5 \mathrm{ml} \mathrm{min}^{-1}\right)$.

$>5$ showed little change. The $\mathrm{CD}$ spectrum of the 1-mer showed a negative peak around $250 \mathrm{~nm}$, and other's showed positive peaks. The intensity in CD spectra was highest on the 3-mer and decreased with an increase in DP accompanying with the shift to longer wave

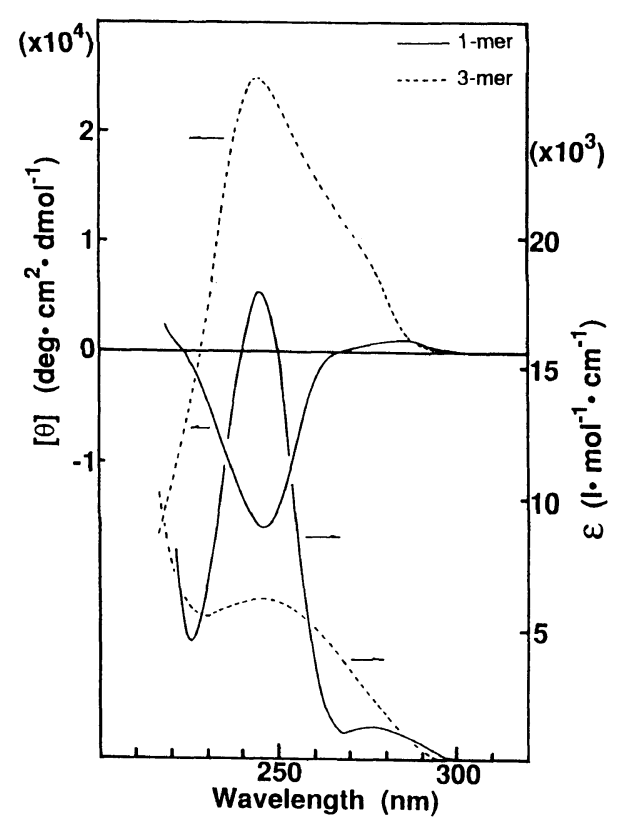

Figure 5. UV and CD spectra of 1-mer and 3-mer in THF.

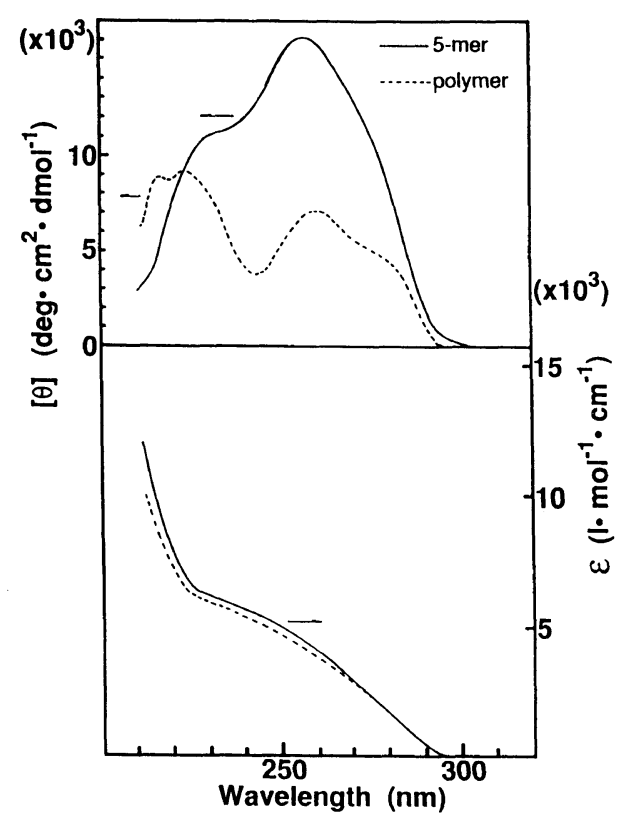

Figure 6. UV and CD spectra of 5-mer and polymer (No. 1 in Table II) in THF.

length.

Table II shows the results of the polymerization at various [monomer]/[initiator] ratios. 
Table II. Polymerization of $m \mathrm{MePI}$ with $\mathrm{Li}-\mathrm{MMP}$ in THF at $-98^{\circ} \mathrm{C}^{\mathrm{a}}$

\begin{tabular}{|c|c|c|c|c|c|}
\hline No. & $\begin{array}{l}{[m \mathrm{MePI}] /} \\
{[\mathrm{Li}-\mathrm{MMP}]}\end{array}$ & $\frac{\text { Yield }^{\mathbf{b}}}{\%}$ & {$[\alpha]_{365}^{25} \mathrm{c}$} & $\begin{array}{l}M_{w}{ }^{d} \\
\left(10^{4}\right)\end{array}$ & $M_{w} / M_{n}{ }^{\mathrm{d}}$ \\
\hline 1 & 50 & 67 & +819 & 1.7 & 1.2 \\
\hline 2 & 100 & 73 & +584 & 2.4 & 1.3 \\
\hline 3 & 200 & 79 & +381 & 3.8 & 1.3 \\
\hline 4 & 500 & 90 & +96 & 7.9 & 1.2 \\
\hline 5 & 1000 & 93 & +29 & 12 & 1.1 \\
\hline
\end{tabular}

a $m$ MePI $0.5 \mathrm{~g}$, THF $5 \mathrm{ml}$, time $4 \mathrm{~h}$.

b Methanol-insoluble part.

c In THF.

d By light scattering method.

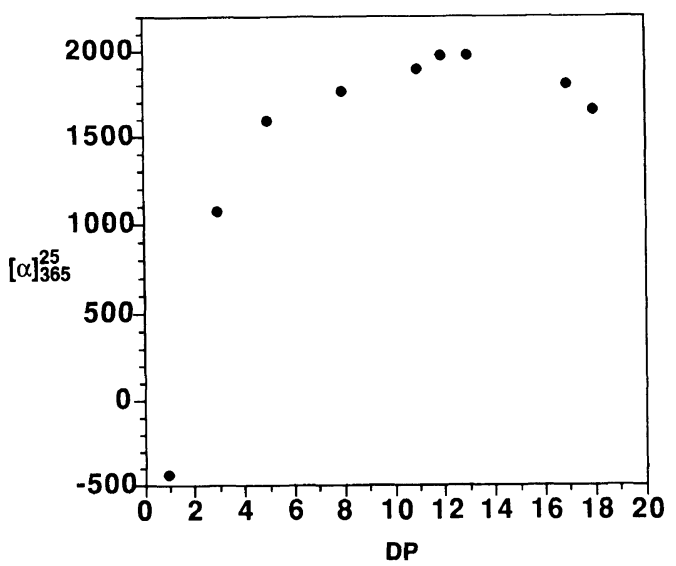

Figure 7. Specific rotation of oligomers of $m \mathrm{MePI}$ in THF.

The optical rotation of the polymer decreased with an increase in molecular weight.

The specific rotation of oligomers is plotted against DP in Figure 7. The specific rotation except for that for 1-mer and 3-mer was estimated from the peak area ratio of the chromatograms monitored with polarimeter and UV detectors as exemplified in Figure 8. The specific rotation $\left([\alpha]_{365}^{25}+1430^{\circ}\right)$ and the peak area ratio (3.0) of oligo-I-4-r, which was isolated as a hexane-ethanol $(4: 1, \mathrm{v} / \mathrm{v})$ insoluble part from oligomer No. 4 in Table I, were selected as the standard.

Figure 9 shows the plots of the specific rotation $v s$. the molecular weight of oligo-

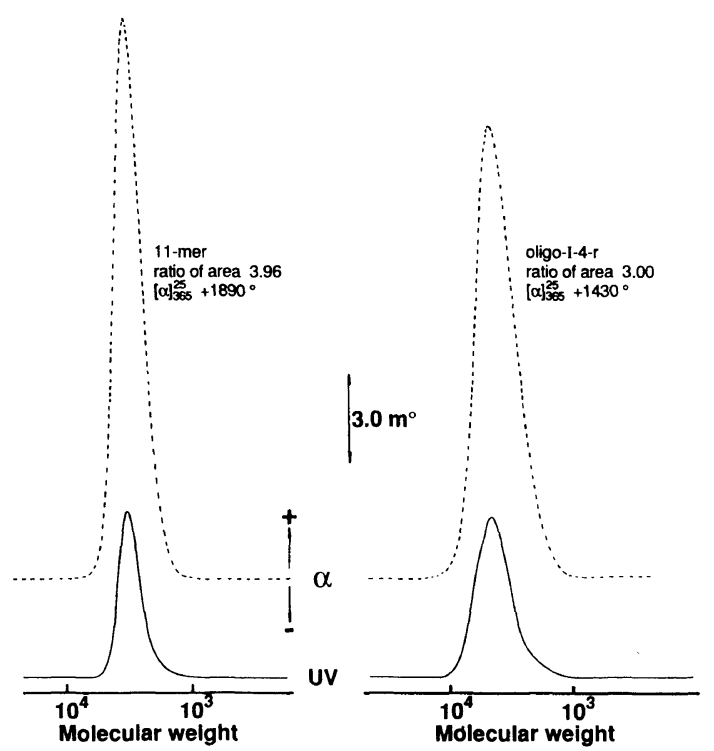

Figure 8. GPC curves of 11-mer and oligo-I-4-r measured with polarimeter and UV $(270 \mathrm{~nm})$ detectors.

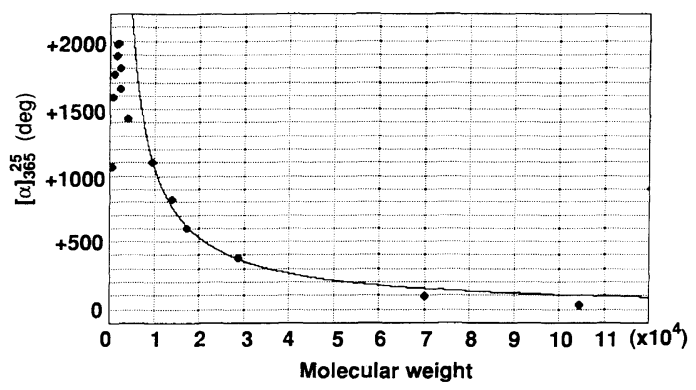

Figure 9. Plots of specific rotation vs. molecular weight of poly $(\mathrm{mMePI})$ in THF.

mers and polymers. The molecular weight of polymers is $M_{n}$ estimated by light scatterig method. Figures 7 and 9 show that the specific rotation increases with an increase in DP in the range of $3 \leq \mathrm{DP} \leq 12$. This is probably because in this DP range the oligomers have a helical structure without a helix reversal point and optical activity increases as the helical structure persists longer with an increase in DP. The gradual decrease of the specific rotation above $\mathrm{DP}=12$ may be ascribed to that the oligomer starts to have helix reversals. Therefore, the polymer chain after a certain 


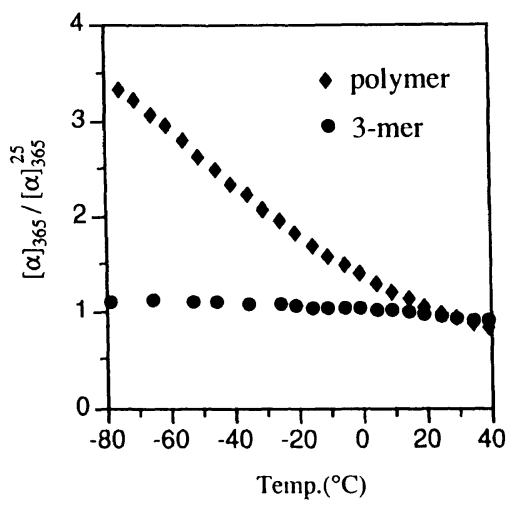

Figure 10. Optical activity of polymer (No. 2 in Table II) and 3-mer in THF at various temperatures.

DP above 12 from $\alpha$-end should have equal amounts of right- and left-handed helical structures which can not contribute to the optical activity. This means that the specific rotation of the polymer will decrease to a half when the molecular weight increases twice. The curve in Figure 9 was obtained by fitting the points of the polymers above molecular weight $9500(\mathrm{DP}=70)$ to the equation $[\alpha]=k / M_{n}$ using a least-squares method. This result shows that the specific rotation is inversely proportional to the molecular weight of the polymer in the range of $D P \geq 70$, but not in the range of $\mathrm{DP} \leq 30$. This probably indicates that the influence of the chiral end group completely disappears at DP $=30-70$.

The specific rotation of the polymer No. 2 in Table II and the 3-mer fractionated by HPLC was measured at various temperatures and the results are shown in Figure 10. The ordinate represents the ratio of the specific rotation at each temperature relative to that at $25^{\circ} \mathrm{C}$. The specific rotation of the polymer increased with a decrease in temperature, but the 3-mer showed almost no change. This may be because at lower temperature the onehanded helical structure originated by a MMP residue can be maintained to higher DP. This should result in the higher optical activity. Such a situation is impossible for the 3-mer.

In this study, we succeeded in the synthesis of the oligomers of an aromatic isocyanate. The chiroptical study of the optically active oligomers with a chiral initiator residue at an $\alpha$-end indicates that the one-handed helical structure starting from the $\alpha$-end may persist at least up to $\mathrm{DP}=12$. This result indicates that in poly(aromatic isocyanate)s helix reversals occur more often than in poly(alkyl isocyanate). ${ }^{7}$ The specific rotation of the polymer with DP $\geq 70$ is inversely proportional to the molecular weight. It was also found that the specific rotation of the polymer was greater at lower temperature because of the increase in the average length of the helical structure of the polymer chain.

Acknowledgment. A part of this study was financially supported by a Grant-in-Aid for Scientific Research (No. 04403021) from the Ministry of Education, Science, and Culture of Japan.

\section{REFERENCES}

1. A. J. Bur and L. J. Fetters, Chem. Rev., 76, 727 (1976).

2. V. E. Shashoua, W. Sweeny, and R. F. Tietz, J. Am. Chem. Soc., 82, 866 (1960).

3. M. Goodman and S. Chen, Macromolecules, 3, 398 (1970).

4. M. Goodman and S. Chen, Macromolecules, 4, 625 (1971).

5. M. M. Green, C. Andreola, B. Munoz, and M. P. Reidy, J. Am. Chem. Soc., 110, 4063 (1988).

6. M. M. Green and M. P. Reidy, J. Am. Chem. Soc., 111, 6452 (1989).

7. S. Lifson, C. Andreola, N. C. Peterson, and M. M. Green, J. Am. Chem. Soc., 111, 8850 (1989).

8. M. M. Green, S. Lifson, and A. Teramoto, Chirality, 3, 285 (1991).

9. Y. Okamoto, M. Matsuda, T. Nakano, and E. Yashima, J. Polym. Sci., A, Polym. Chem., 32, 309 (1994).

10. Y. Okamoto, M. Matsuda, T. Nakano, and E. Yashima, Polym. J., 25, 391 (1993). 